\title{
High Capacity Color Barcodes Using Dot Orientation and Color Separability
}

\author{
Orhan Bulan $^{a}$, Vishal Monga ${ }^{b}$, Gaurav Sharma ${ }^{a}$ \\ ${ }^{a}$ University of Rochester, Rochester, NY, USA \\ ${ }^{b}$ Xerox Corporation, Webster, NY, USA
}

\begin{abstract}
Barcodes are widely utilized for embedding data in printed format to provide automated identification and tracking capabilities in a number of applications. In these applications, it is desirable to maximize the number of bits embedded per unit print area in order to either reduce the area requirements of the barcodes or to offer an increased payload, which in turn enlarges the class of applications for these barcodes. In this paper, we present a new high capacity color barcode. Our method operates by embedding independent data in two different printer colorant channels via halftone-dot orientation modulation. In the print, the dots of the two colorants occupy the same spatial region. At the detector, however, by using the complementary sensor channels to estimate the colorant channels we can recover the data in each individual colorant channel. The method therefore (approximately) doubles the capacity of encoding methods based on a single colorant channel and provides an embedding rate that is higher than other known barcode alternatives. The effectiveness of the proposed technique is demonstrated by experiments conducted on Xerographic printers. Data embedded at a high density by using the two cyan and yellow colorant channels for halftone dot orientation modulation is successfully recovered by using the red and blue channels for the detection, with an overall symbol error rate that is quite small.
\end{abstract}

Keywords: H ardcopy data coding, color barcodes, orientation modulation, color separation

\section{INTRODUCTION}

Embedding information in printed documents continues to be a problem of considerable interest in security applications. A large number of legal and official documents such as IDs, passports, and other transactional data are consumed in the printed format. Hence, systems and algorithms for protecting and interacting with hardcopy content are a necessity. In this context, techniques for embedding and extracting digital data in hardcopy documents, which are fundamentally analog, are of particular interest because these methods can add security and functionality and features that are associated with digital techniques, for example by enabling cryptographic constructs for authentication, data integrity verification, non-repudiability, etc. ${ }^{11,12}$ Methods for digitally embedding and extracting data in hardcopy documents can be classified as either data hiding or data encoding methods depending, respectively, on whether the data is imperceptibility inserted in a cover document whose primary function is other than the communication of the data or whether a region of the document is devoted solely to the objective of communicating the embedded data. Methods in both classes are of interest for a variety of hardcopy security applications and in the literature a number of techniques have been reported for data hiding ${ }^{3-10}$ and for data encoding. ${ }^{1,2,17}$ In this paper, we focus on 2-D barcodes, a specific technique within the latter category.

Two-dimensional (2-D) barcodes are widely utilized for data encoding in hardcopy documents. ${ }^{1,2}$ Besides being convenient and cheap, they offer greater capacity for data embedding than their one-dimensional counterparts and also in comparison with hardcopy data hiding techniques. The increased capacity can often enable

Send correspondence to O. Bulan: E-mail: bulan@ece.rochester.edu, Telephone: 1585 275-8122, Address: Electrical and Computer Engineering Department, University of Rochester, Rochester, NY, 14627-0126, USA, WWW: www.ece.rochester.edu/projects/iplab

This work was supported in part by Xerox Corporation and by the a grant from New York State Office of Science, Technology and Academic Research (NYSTAR) through the Center for Electronic Imaging Systems (CEIS).

Media Forensics and Security, edited by Edward J. Delp III, Jana Dittmann,

Nasir D. Memon, Ping Wah Wong, Proc. of SPIE-IS\&T Electronic Imaging, SPIE Vol. 7254,

$725417 \cdot$ ? 2009 SPIE-IS\&T · CCC code: 0277-786X/09/\$18 · doi: 10.1117/12.807742

SPIE-IS\&T/ Vol. 7254 725417-1 
additional security applications. For instance, in personal ID's, driving licenses and passports, this additional capacity can enable the embedding of a sample speech signal, a picture, or other identifying personal information which in turn may be used to establish authenticity of the printed document and/or the identity of the user. Particular examples proposed in the literature include identification cards based on biometrics ${ }^{13}$ and print signatures for document authentication ${ }^{14}$ ). Several of these applications favor a push toward higher data rates for two-dimensional codes, which is the specific aspect that we address in our work.

Many of the existing 2-D barcodes in the literature, are based on monochromatic and single ink printing. The extension to color is highly desirable for the benefits it carries in terms of embedding rate per unit area and hence, to meet the requirements of cryptographic protocol and several new applications. To the best of our knowledge, there are two known color barcode schemes: one recently developed at Microsoft ${ }^{15,16}$ and the other being DataGlyphs. ${ }^{17,18}$ The former method encodes the data as triangles in one of four colors (black, red, green and yellow) where the color is chosen based on the data. While this allows for each triangle to carry a 4 - ary value, as we see subsequently, the flexibility afforded by the spectral difference between the colorants is not fully exploited. DataGlyphs, on the other hand, in their color instantiation operate by simply using the same glyph pattern in a dot-on-dot mode that fundamentally offer the same capacity as a single channel.

In this paper, we propose a new high capacity color barcode technique by exploiting halftone-dot orientation and color separability. In previous work, we developed a hardcopy data hiding technique ${ }^{10,19}$ that uses elliptically shaped halftone dots and embeds data in the choice of the ellipse orientation. Here we extend that method to embedding using color halftone dots. In particular, we exploit the spectral (wavelength) characteristics of the Cyan, Magenta, Yellow colorants commonly used in digital printing, and those of Red, Green and Blue sensors widely used in desktop scanners. We demonstrate that carefully designed mixtures of two colorants can actually be well-separated from the scan and hence result in double the capacity of a embedding method that works on a single colorant channel. We demonstrate experimentally that our method can achieve the highest embedding rates of all hardcopy barcodes known in the literature.

The rest of this paper is organized as follows. Section 2 gives an overview of data embedding via halftone-dot orientation modulation. In Section 3 we first introduce color separability and then describe the proposed data coding method. We present the preliminary results in Section 4 . Section 5 concludes the paper by summarizing the key aspects of our scheme.

\section{DATA EMBEDDING VIA HALFTONE-DOT ORIENTATION MODULATION}

In recent work, we developed a data hiding method for hardcopy images via halftone dot orientation modulation. ${ }^{10}$ This method specifically offers the ability to generate elliptically shaped halftone dots with orientation control. Data embedding is then carried out in a particular choice of orientation. Figure 1 shows a zoomed version of a constant graylevel image where halftone dots are oriented along vertical, horizontal and diagonal $(+/-45$ degree) directions, i.e. each orientation may be thought of as representing a binary symbol.

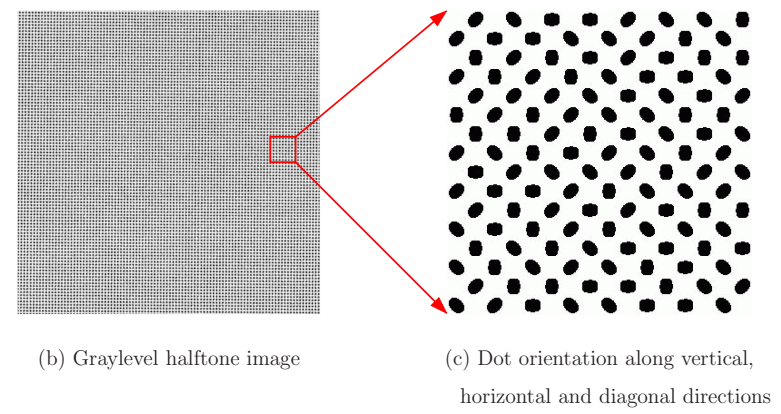

Figure 1. Orientation based data embedding

At the receiver, first the scan is synchronized both for global affine transforms, and local variations. ${ }^{10}$ Once a halftone cell is identified in the (scanned) synchronized image, detection is based on $M$ statistical moments, each corresponding to an ellipse orientation. For example, the moment along $Y$-axis is calculated as: 


$$
\sigma_{1}=\frac{1}{A} \sum_{x, y \in C} I^{s}(x, y)(y-\bar{y})^{2}
$$

where $A=\sum_{x, y \in C} I^{s}(x, y)$ and $\bar{y}=\frac{1}{A} \sum_{x, y \in C} I^{s}(x, y) y$ represents the co-ordinate of the center of mass of the halftone dot. The remaining $M-1$ moments $\sigma_{i}, \quad i=2,3 \cdots,(M-1)$ can be computed similarly.

\section{COLOR BARCODES USING DOT ORIENTATION AND COLOR SEPARABILITY}

\subsection{Color Separation}

Color devices exhibit considerable diversity in their color spaces. While color is physically a continuous stimulus, devices typically use a lower-dimensional (3-D/4-D) representation of color. Printing systems are mostly based on cyan, magenta, and yellow (CMY) colorants, while displays and capture devices such as scanners, digital cameras use a color model based on red, green, and blue (RGB) phosphors or sensors to represent color images. Based on the utilization of RGB or CMY spaces, color devices can be categorized as additive or subtractive. ${ }^{20}$

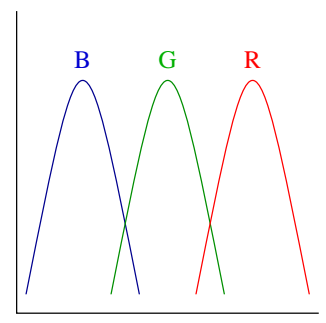

Wavelength

(a)

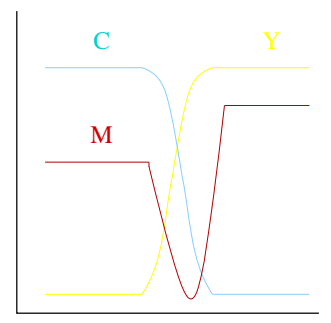

Wavelength

(b)

Figure 2. Spectra of RGB and CMY colorants

Additive color systems such as cameras and scanners represent color through the combination of additive red, green, and blue primaries. The additive mixing of two primaries, forms cyan, magenta and yellow while the combination of all three gives white and the absence of all results in black. Subtractive systems, on the other hand, use CMY space and form color by subtracting unwanted spectral components from the white light. In particular, cyan absorbs the spectral region corresponding to red sensation, magenta absorbs the region corresponding to green and yellow eliminates blue. Figure 2 (a) shows the spectral emissions of RGB lights and Fig. 2 (b) denotes the spectral transmittance of CMY colorants where each absorbs in one region of the spectrum.
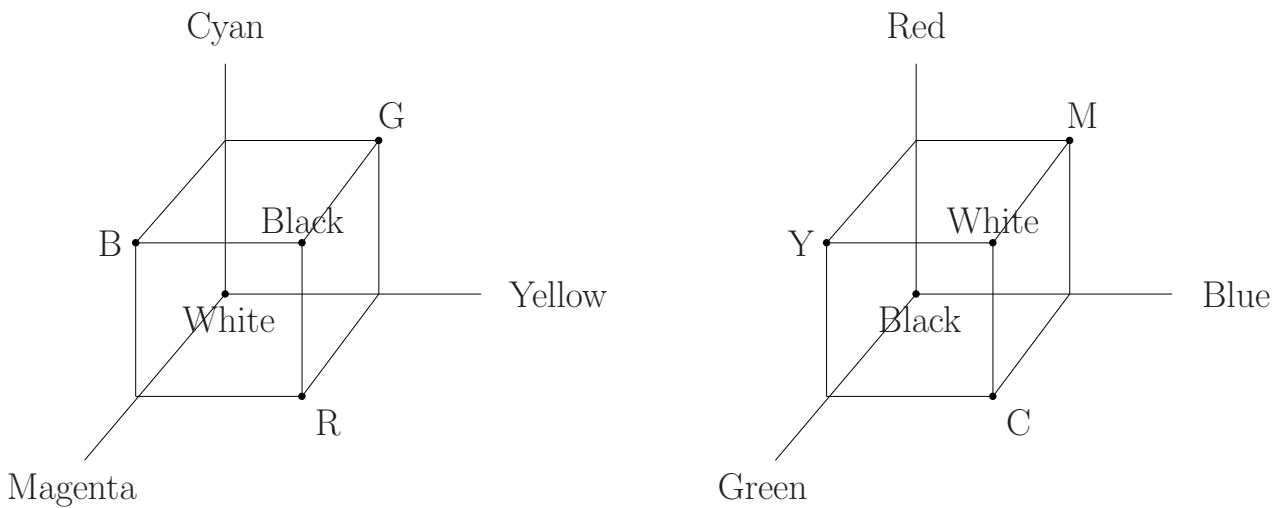

Green

Figure 3. CMY and RGB color cubes 
Note that based on their spectral characteristics, colorants in CMY space are complementary to RGB colorants respectively. Fig. 3 shows idealized CMY and RGB color cubes to illustrate this point.
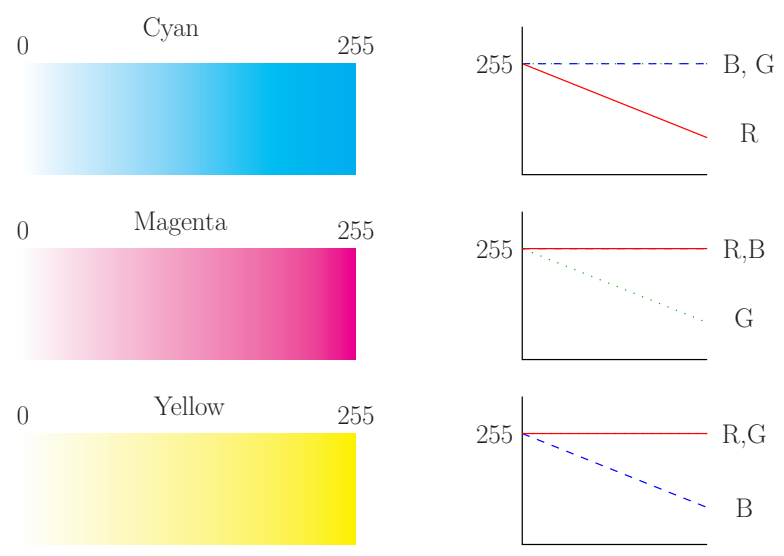

Figure 4. Complementary colors

\subsection{Color Barcodes}

We exploit this complementary relation between CMY and RGB colorants for creating color barcodes. The underlying principle of color separability is illustrated in Fig. 4 for an idealized color printing and scanning system. In the figure, we generate ramp functions ranging from zero color level to full color level (digital value 255 in typical 8-bit representation) for each of the $\mathrm{C}, \mathrm{M}$, and Y colorants. Corresponding to each ramp, we plot the variation in the RGB channels as observed by an idealized scanner. Note that in this idealized scenario, the CMY colorants only absorb the light energy in non-overlapping wavelength bands and therefore do not interfere with bands corresponding to other colorants. The red channel can hence convey information about the Cyan colorant, the green for Magenta, and blue for Yellow.

Similar plots for two color ramps are shown in Fig. 5. Note in the idealized case, the two complementary channels can be used to separate the mixture of two colorants. In practice, however as revealed in Fig. 2 the absorption bands of individual colorants interfere with each other and cause unwanted absorptions in the spectra of the color print. We therefore, select cyan and yellow channels to embed data since their absorption bands in the spectra are the most distant from each other as shown in Fig. 2 (b).

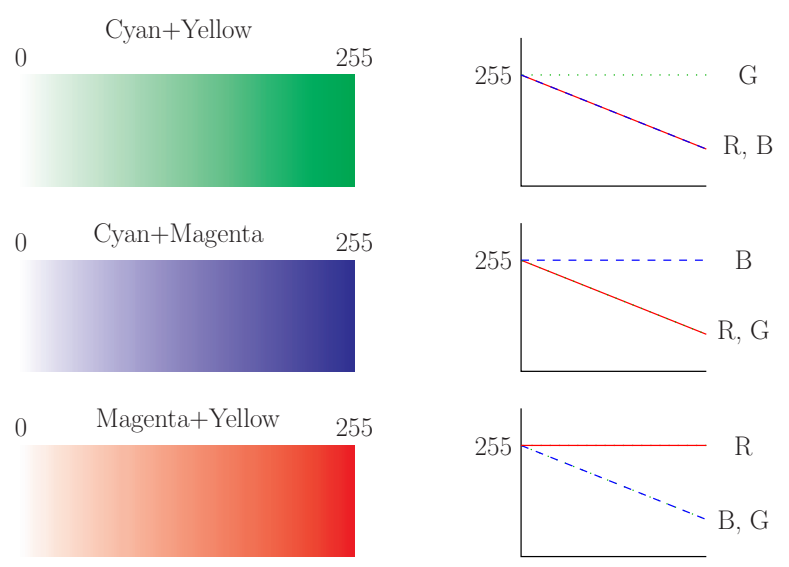

Figure 5. Two color mixture

In each selected color channel i.e. cyan and yellow, we then embed data by halftone-dot orientation modulation described in Sec. 2. The data embedding capacity offered by the orientation modulation technique exhibits a 
considerable variation* ${ }^{*}$ with the digital level i.e. area coverage for the colorant. ${ }^{21}$ We therefore, determine the input digital level for each colorant at which our modulation technique offers the highest capacity. We then generate the halftoned barcode bearing the embedded data with the pre-determined color levels. Figure 6(a) shows the proposed digital barcode with 4 - ary modulation.

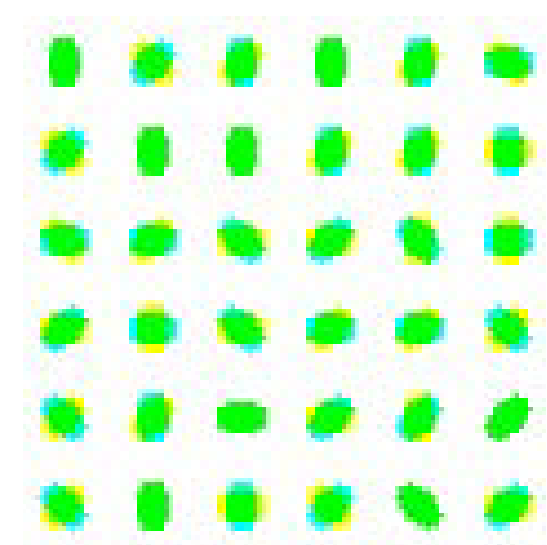

(a) Color Barcode via halftone dot orientation modulation

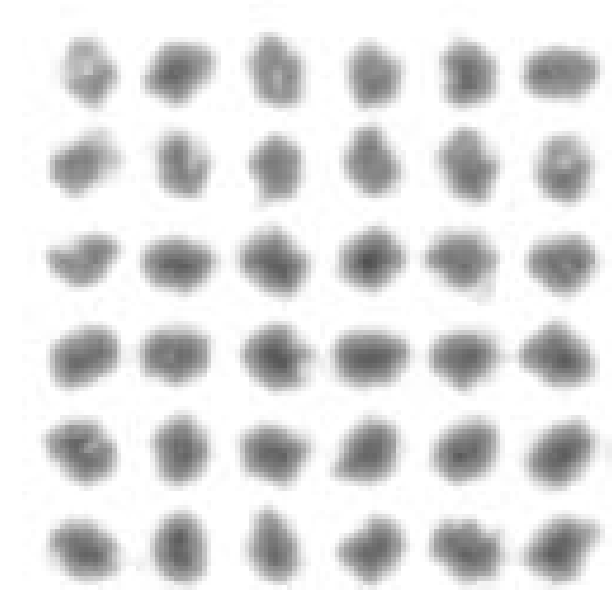

(c) Separated cyan from the scanned barcode (Red separation)

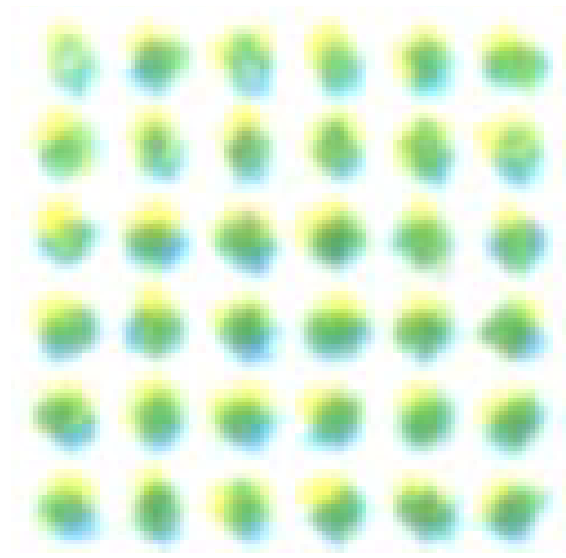

(b) Scanned Color Barcode

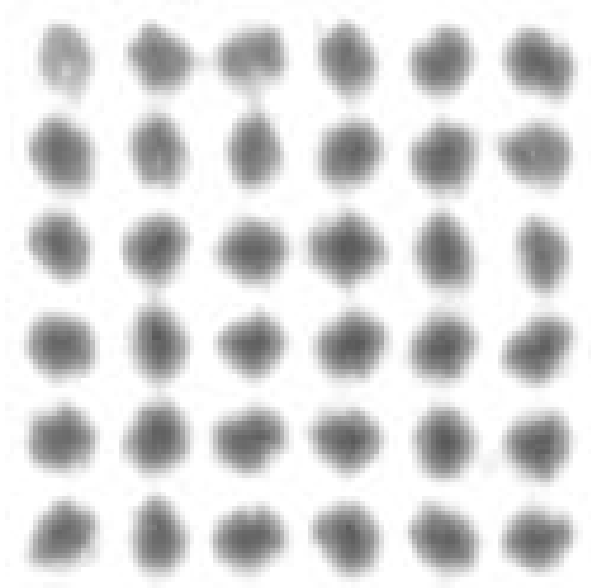

(d) Separated yellow from the scanned barcode (Blue separation)

Figure 6.

\section{EXPERIMENTAL RESULTS}

We evaluate the performance of the proposed color barcodes experimentally by utilizing a xerographic printer and a flatbed scanner. In our experiments, we perform dot-on-dot halftoning and utilize $0 / 90^{\circ}$ orthogonal CY halftone screens with a frequency of 120 cells per inch (cpi) to generate the halftone barcode. The resulting digital barcode is printed on a xerographic color printer with an addressability of 2400 dots per inch (dpi). The resulting print is then scanned on a flatbed scanner and colors are separated from the scanned image. Figure 6 (c) and (d) illustrates the separated cyan and yellow colorants from the scanned barcode shown in Fig. 6 (b). Following

\footnotetext{
${ }^{*}$ This variation is identical to the variation in monochrome images, which has been studied in our prior work. ${ }^{21}$
} 
color separation, moments are extracted from each individual color channel and orientation is estimated based on the extracted moments.

In Table 1, we list the symbol error rate (SER) performance of the proposed color barcode method. We evaluate the performance for both binary and 4-ary orientation modulation with scan resolution values of 1200 dpi and 2400 dpi. While we can embed 28,800 bits per inch square via binary modulation with SER less than $5 \times 10^{-3}, 4$ - ary modulation allows to embed 57,600 bits per inch square with SER less than $6 \times 10^{-2}$. Here, we only report the SER performance of the proposed technique, but the error free operational rates can also be obtained by utilizing error correction coding as has been demonstrated in our recent work in data hiding in monochrome images. ${ }^{19}$ Comparing with the high capacity Microsoft color barcodes that can embed 16,000 bits per square inch, we estimate that the proposed method can achieve embedding rates that are higher than all existing barcodes. Our technology is largely robust against inter-separation mis-registration since we embed and detect in each separation independently whereas the Microsoft technology is sensitive to misregistration since they require their triangles to be the correct colors. The colors however will change with misregistration especially in high resolution printing that enables higher embedding rates.

\begin{tabular}{|c|c|c|c|}
\hline Modulation & Scanner Res.(dpi) & SER & Bits/inch sq. \\
\hline Binary & 1200 & 0.0201 & 28,800 \\
\hline Binary & 2400 & 0.0047 & 28,800 \\
\hline $4-$ ary & 1200 & 0.1316 & 57,600 \\
\hline $4-$ ary & 2400 & 0.0559 & 57,600 \\
\hline
\end{tabular}

Table 1.

\section{CONCLUSION}

We construct a high capacity color barcode by extending our previously proposed hardcopy data hiding method based on halftone dot orientation modulation. The fundamental underlying principle of our work is that two-color mixtures of the Cyan and Yellow colorant channels used widely in digital printers can be well separated using the red and blue channels of common desktop RGB scanners owing to their complementary spectral characteristics. By exploiting this separability, our method enables printing of two colors at the same spatial location, and hence (nearly) doubles the capacity in comparison with of encoding methods based on a single colorant channel. Preliminary experiments conducted on Xerographic printers show that our method can significantly outperform the state of the art in 2-D barcodes achieving a higher embedding rate.

\section{REFERENCES}

1. N. Damera-Venkata, J. Yen, V. Monga, and B. Evans, "Hardcopy image barcodes via block-error diffusion," IEEE Trans. Image Proc. 14, pp. 1977-1989, Dec. 2005.

2. R. Villán, S. Voloshynovskiy, O. Koval, and T. Pun, "Multilevel 2d bar codes: Towards high capacity storage modules for multimedia security and management," IEEE Transactions on Information Forensics and Security 1, pp. 405-420, December 2006.

3. D. Kacker and J. P. Allebach, "Joint halftoning and watermarking," Signal Processing, pp. 1054-1068, Apr. 2003.

4. K. T. Knox and S. Wang, "Digital watermarks using stochastic screens," in Proc. SPIE: Color Imaging: Device Independent Color, Color Hardcopy, and Graphic Arts II, G. B. Beretta and R. Eschbach, eds., 3018, pp. 316-322, Feb. 1997.

5. S. Wang, "Digital watermarking using phase-shifted stoclustic screens." US Pat. \#6,252,971, 2001.

6. G. Sharma and S. Wang, "Show-through watermarking of duplex printed documents," in Proc. SPIE: Security, Steganography, and Watermarking of Multimedia Contents VI, E. J. Delp and P. W. Wong, eds., 5306, pp. 670-684, Jan. 2004.

7. B. Oztan and G. Sharma, "Continuous phase modulated halftones and their application to halftone data embedding," in Proc. IEEE Intl. Conf. Acoustics Speech and Sig. Proc., II, pp. 333-336, May 2006. 
8. D. Corp, "Digimarc mediabridge." 2000.

9. K. Solanki, U. Madhow, B. S. Manjunath, S. Chandrasekaran, and I. El-Khalil, "Print and scan' resilient data hiding in images," IEEE Trans. Info. Forensics and Security 1, pp. 464-478, Dec. 2006.

10. O. Bulan, V. Monga, G. Sharma, and B. Oztan, "Data embedding in hardcopy images via halftone-dot orientation modulation," in Proc. SPIE: Security, Forensics, Steganography, and Watermarking of Multimedia Contents X, E. J. Delp, P. W. Wong, J. Dittmann, and N. D. Memon, eds., 6819, pp. 68190C-1-12, Jan. 2008.

11. A. Menezes, P. van Oorschot, and S. Vanstone, Handbook of Applied Cryptography, CRC Press, Florida, USA, 1997.

12. D. Stinson, Cryptography: Theory and Practice, CRC Press, Florida, USA, second ed., 2002.

13. D. Schonberg and D. Kirovski, "EyeCerts," IEEE Transactions on Information Forensics and Security $\mathbf{1}(2)$, pp. 144-153, 2006.

14. B. Zhu, J. Wu, and M. S. Kankanhalli, "Print signatures for document authentication," in Proceedings CCS'03, ACM, 2003.

15. "http://research.microsoft.com/research/hccb/about.aspx." accessed July 2008.

16. D. Parikh and G. Jancke, "Localization and Segmentation of A 2D High Capacity Color Barcode," Applications of Computer Vision, 2008. WACV 2008. IEEE Workshop on, pp. 1-6, 2008.

17. D. L. Hecht, "Printed embedded data graphical user interfaces," IEEE Computer , pp. 47-55, 2001.

18. D. L. Hecht, "Embedded data glyph technology for hardcopy digital documents," in Proc. SPIE: Color hard copy and graphic arts III, J. Bares, ed., 2171, pp. 341-352, Mar. 2001.

19. O. Bulan, G. Sharma, and V. Monga, "Adaptive decoding for halftone orientation-based data hiding," in Proc. IEEE Intl. Conf. Image Proc., pp. 1280-1283, Oct. 2008.

20. G. Sharma, ed., Digital Color Imaging Handbook, CRC Press, Boca Raton, FL, 2003.

21. O. Bulan, G. Sharma, and V. Monga, "On the capacity of orientation modulation halftone channels," in Proc. IEEE Intl. Conf. Acoustics Speech and Sig. Proc., pp. 1685-1688, (Las Vegas, Nevada), Apr. 2008. 